\title{
Hepatitis A, B y D en Chocó
}

\author{
Julio C. Padilla ${ }^{1}$, Ana L. Arriaga ${ }^{2}$
}

\section{Resumen}

Se realiza una recopilación y análisis de la información epidemiológica existente sobre hepatitis A, B y delta en el departamento de Chocó en el período 1976-1993. En un estudio anterior, se informó una prevalencia de anticuerpos contra el virus de la hepatitis A de 85,7 por mil, en una muestra representativa de la población, con evidencia de transmisión en todos los grupos de edad; en la misma muestra, se encontró una prevalencia de antígeno de superficie de hepatitis B de 4,2/1.000.

Se han detectado focos de hepatitis fulminante aguda por hepatitis delta en la región de Truandó-Salaquí, en el municipio de Riosucio, donde se encontró una prevalencia de infección por hepatitis B de 915/1.000 y una prevalencia de antígeno de superficie de hepatitis B de 200/1.000. El principal patrón de transmisión es vertical-horizontal. Los principales factores de riesgo de importancia en la transmisión en esa área son el hacinamiento, la promiscuidad, los grupos femeninos de 15 a 24 años y la evidencia de transmisión en algún miembro de la familia.

En la zona rural de Quibdó, se han identificado dos focos de hepatitis fulminante aguda en las localidades de Bebaramá y Guarandó, encontrándose prevalencias de infección por hepatitis B de 74 y $85 \%$.

En un estudio realizado en personal de salud cuyo objetivo era identificar los grupos con mayor riesgo, se encontraron prevalencias de infección entre 27 y $33 \%$; además, la prevalencia de antígeno de superficie de hepatitis B fue 13,3 a $13,6 \%$, principalmente en personal de enfermería y laboratorio.

Finalmente, se plantean alternativas integrales de solución para hepatitis A y $\mathrm{B}$ basadas en actividades de promoción de la salud, medidas de prevención como la vacunación contra hepatitis $\mathrm{B}$, la vigilancia y el control.

\section{Summary}

A study to compile and analyse the existing epidemiological information concerning hepatitis A, B and D in the Chocó department of Colombia was carried out from 1976-1993. One study reported a hepatitis A virus antibody prevalence of 85.7 per thousand, in a representative population sample. There was evidence of transmission amongst all age groups. In the same sample a hepatitis B surface antigen prevalence of 4.2 per thousand was found.

Acute fulminant hepatitis foci for hepatitis $D$ have been detected in the town of Riosucio in the Truandó-Salaquí region, where hepatitis B infection prevalence of 915 per thousand

\footnotetext{
${ }^{1}$ Ministerio de Salud, Santa Fe de Bogotá.

${ }^{2}$ Oficina de Servicio Seccional de Salud del Chocó, Quibdó.
} 
and 200 per thousand hepatitis B surface antigen prevalence was found. The main transmission axis is vertical-horizontal. The principal important transmission risk factors in this area are: overcrowding, promiscuity, the 15-24 female age group and the evidence of transmission in any family member.

Two foci of acute fulminant hepatitis have been identified in Bebaramá and Guarandó in the rural zone of Quibdó, hepatitis B infection prevalencies of $74 \%$ and $85 \%$ having been found.

A study carried out with health personnel, whose objective was to identify groups most at risk, found infection prevalencies between $27 \%$ and $33 \%$. Furthermore, hepatitis B surface antigen prevalence was $13.3 \%$ and $13.6 \%$, principally in nursing and laboratory personnel.

Finally, integral solution alternatives were planned for hepatitis $A$ and $B$ based on health promotion activities, preventative measures (such as hepatitis B vaccination), vigilance and control.

Las hepatitis virales son uno de los mayores problemas de salud pública en muchas partes del mundo. La distribución de la hepatitis A es mundial; se presenta en forma esporádica y epidémica. En los países en desarrollo, los adultos suelen ser inmunes y son raras las epidemias de hepatitis A. Mundialmente, las áreas geográficas se pueden caracterizar como de alta, mediana y baja endemicidad, de acuerdo con las infecciones por HVA, las edades usuales de infección, los grupos en riesgo y la aparición de brotes $(1,2)$.

Las infecciones por hepatitis $B$ afectan alrededor de 2.000 millones de personas a nivel mundial y ocurren 2 millones de muertes al año por esta causa. Además, existen cerca de 300 millones de portadores crónicos, $25 \%$ de los cuales morirá por enfermedad crónica o hepatocarcinoma (3).

En América Latina y el Caribe, se calculan cerca de 5 millones de portadores de HBsAg y más de 400.000 infecciones nuevas cada año (4).

La hepatitis delta es de distribución mundial, pero su prevalencia varía ampliamente. Se presenta en forma epidémica o endémica en grupos de población en alto riesgo de adquirir la infección por el VHB. Es causada por co o sobreinfección del virus delta en portadores de hepatitis $B$ y es una grave amenaza para niños y adultos jóvenes de zonas deprimidas. En las Américas, se han registrado brotes epidémicos graves en países como Brasil, Venezuela y Colombia (1).

En Colombia, la infección por virus de la hepautitis By hepatitis fulminante han sido reconocidas como endémicas en varias regiones como el pie de monte de la Sierra Nevada de Santa Marta, la Serranía del Perijá, el Urabá chocoano y algunos sitios del trapecio amazónico (5-8).

Las hepatitis víricas A, B y D representan un grave problema de salud en la población chocoana por su alta endemicidad y epidemicidad en varias áreas del departamento. Las infecciones por hepatitis A afectan a todos los grupos de edad y su prevalencia es de 875/1.000; la prevalencia general de portadores de antígeno de superficie de hepatitis B se estima en 4,2/1.000, pero existen áreas de Riosucio y Quibdó con prevalencias entre 74 y $92 \%$. Igualmente, existen focos estudiados de hepatitis $D$ en la región Atrato-Truandó y en la zona rural de Quibdó (9).

El objetivo del presente estudio es realizar una aproximación de la magnitud real del problema que representan las hepatitis víricas A, B y D en Chocó, a partir de la información existente con el propósito de recomendar e implementar medidas de prevención y control sostenidas que impacten los riesgos y la morbimortalidad ocasionada por estas enfermedades.

\section{Materiales y métodos}

Se realiza una descripción del comportamiento epidemiológico de las hepatitis A, B y D durante el período comprendido entre 1976 y 1993. Se hizo una recopilación y análisis de la información epidemiológica existente, utilizando fuentes secundarias como el registro de mortalidad del DANE, el registro de enfermedades de notificación obli- 
gatorias o SIS-12, y las investigaciones y estudios de brotes realizadas en el período estudiado.

Los criterios de casos utilizados en los diferentes estudios diferentes de morbilidad y de brotes fueron: infectados eran las personas positivas a cualquier marcador serológico; inmunes los que tenían positivos antiHBc y antiHBs; con infección B activa se consideraron aquellas personas con AgsHVB positivo, antiAgsHBV negativo y antiHVBc negativo; portadores de antígeno de superficie de hepatitis $B$ eran todas las personas con positividad al HBsAg, y susceptibles eran todos los seronegativos a todos los marcadores utilizados.

En la investigación del brote de hepatitis fulminante, se consideró como coinfección HBsA los que presentaban $\mathrm{HBsAg}$ positivo, antiHBcAg positivo y antidelta positivo y sobreinfección, $\mathrm{HBsAg}$ positivo y antidelta positivo.

Las determinaciones se realizaron utilizando la técnica de Elisa.

En el brote de hepatitis fulminante en Riosucio, se estudiaron 492 personas en las localidades de Bocas de Taparal, Chintadó, La Nueva, Quiparadó, Truandó, Domingodó, Curvaradó, Topogadó y Río Ciego. Se realizó el estudio de marcadores serológicos como antígeno de superficie de hepatitis $\mathrm{B}$, anticore $\lg \mathrm{G}$ e $\lg \mathrm{M}$, anticuerpos contra el antígeno de superficie de hepatitis $B$ y anticuerpos contra el virus de la hepatitis delta.

En el estudio de brote de la localidad de Bebaramá, municipio de Quibdó, con una población de 127 personas, se realizó una encuesta serológica del $60 \%$ de los habitantes para determinar marcadores de hepatitis B como AgsHBV y antiHVBc. Los casos fatales fueron comprobados mediante estudio histopatológico postmortem.

Para determinar la prevalencia de infección por hepatitis B en la población del municipio de Riosucio y establecer los principales factores de riesgo asociados en la transmisión de la HVB, se realizó, en 1992, un estudio seroepidemiológico de marcadores de hepatitis $B$ y factores de riesgo. Se calculó una muestra aleatoria mínima de 912 personas, con $95 \%$ de confianza, esperando una prevalencia de infección de $65 \%$, mediante un muestreo por conglomerados polietápico, to- mando como congiomerados las áreas geográficas, las localidades y las viviendas, muestreándose finalmente los voluntarios de acuerdo con la distribucón de la población general de Riosucio.

Los marcadores de hepatitis $B$ que se realizaron fueron: antígeno de superficie de hepatitis $B$, anticuerpo contra el antígeno de superficie de HVB y anticore; las muestras recolectadas fueron enviadas refrigeradas al Instituto Nacional de Salud, donde se realizaron pruebas de Elisa para la determinación de los marcadores. A los participantes en el estudio se les aplicó un instrumento que recolectaba las variables para determinar factores de riesgo.

En Guarandó, municipio de Quibdó, se realizó una investigación de brote donde se hizo una encuesta serológica en 78 personas para determinar la prevalencia de infección por HVB en la población general y de convivientes para implementar medidas de prevención y control.

En 1993, con el objetivo de establecer las categorías de infección en los trabajadores de la salud con exposición ocupacional a la HVB, se realizó una encuesta seroepidemiológica en los hospitales regionales de Quibdó e Itsmina.

Se seleccionó una muestra de trabajadores $(n=249)$ en riesgo ocupacional para hepatitis $B$, esperando una prevalencia de infección de $20 \%$, con una confianza de $95 \%$, mediante muestreo por conglomerados, bietápico y estratificado por región, obteniéndose 153 para la región del Atrato y 93, para el San Juan.

\section{Resultados}

De acuerdo con el SIS-12, se han notificado 802 casos de hepatitis víricas en general, sin especificación por tipo, en el departamento de Chocó, en el período estudiado, con una proporción de prevalencia mediana de 15,7/100.000 hab, registrándose las frecuencias más altas, 33,8, en 1983 y las más bajas en 1979 y 1982 . El $77,6 \%$ de los casos notificados en el período se registraron en los municipios de Quibdó, Carmen de Atrato, Istmina, Riosucio, San José del Palmar y Acandí (10).

En el estudio de morbilidad realizado por Engelkes et al., se encontró una proporción de prevalencia 
de anticuerpos para HVA de 875/1.000. Se observó que había transmisión en todos los grupos de edad (figura 1).

En esa muestra se encontró una seroprevalencia de antígeno de superficie de hepatitis B de 4,2/ 1.000. En la figura 2, se observa que en la distribución por grupos de edad se encontró que los menores de un año tenían una prevalencia de AgsHBV de 74/1.000 y de $70 / 1.000$ en el grupo de 25 a 44 años (figura 2).

Los resultados de la investigación del brote de hepatitis fulminante en Riosucio registraron una mortalidad cercana a 25 personas, pero sólo se logró determinar la muerte de ocho, cuatro por estudio histopatológico postmortem con cuadro de necrosis masiva y submasiva.

La prevalencia de inmunes encontrada fue de $83 \%$, $11 \%$ de portadores de antígeno de superficie de hepatitis B y $6 \%$ de infección activa. De 18 posi-

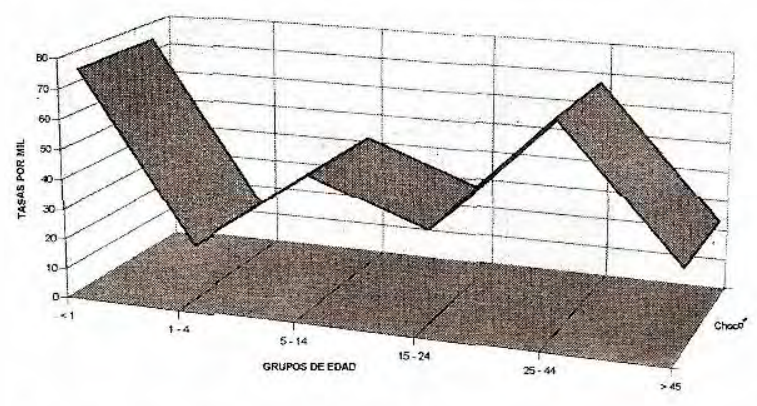

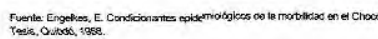

Figura 1. Distribución de antígenos de hepatitis B por grupos de edad, Chocó, 1986.

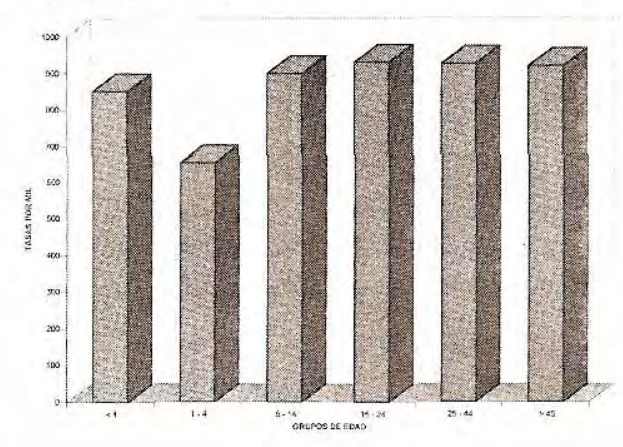

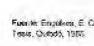

Figura 2. Distribución de anticuerpos de hepatitis A por grupos de edad, Chocó, 1986. tivos a HVD, se encontraron 9 inmunes, 3 casos de sobreinfección y 1 con infección delta por serología de marcadores de hepatitis delta y $\mathrm{B}$. En la localidad de Chintadó, se encontró que 29\% de las personas tenía evidencia de coinfección delta y $50 \%$ para hepatitis B. Los convivientes de casos fatales presentaron un riesgo 13 veces mayor para contraer hepatitis delta que los no convivientes (12).

En el brote ocurrido en la localidad de Babaramá, en agosto de 1990, se registraron 2 casos fatales de hepatitis fulminante aguda en adolescentes, con cuadro clínico de aparición brusca y curso rápido caracterizado por ictericia, adinamia, vómitos, melenas, oliguria, convulsiones y muerte. Estos fueron comprobados por estudio histopatológico postmortem que mostró un cuadro de necrosis hepática submasiva con células moruliformes. Se encontró una prevalencia de infección global de hepatitis $\mathrm{B}$ de $74 \%$ y una proporción de prevalencia de antígeno de superficie de $7 \%$. La mayor frecuencia se encontró entre los grupos de 10 a 19 años (13).

La investigación de seroprevalencia de infección por hepatitis $B$ y factores de riesgo en Riosucio mostró una prevalencia global de infección por hepatitis $\mathrm{B}$ en la población de $85 \%$ y una proporción de prevalencia de antígeno de superficie de hepatitis $\mathrm{B}$ de $20 \%$. El cuadro 1 muestra la distribución de HVB por categorías y zonas; la prevalencia en el área Truandó-Salaquí fue de 91,5\% mientras que el área Riosucio-LalargaJiguamiandó fue de $72,4 \%$.

\section{Discusión}

Chocó es un área de alta prevalencia de infección por hepatitis $\mathrm{A}$, siendo el riesgo de adquirir la enfermedad similar en los diferentes grupos de edad. Las condiciones de vida de la mayoría de la población del Chocó se han caracterizado por altas necesidades básicas insatisfechas en servicios básicos, deficiente saneamiento del medio, utilización común de fuentes de agua para ingerir, descarga fecal, lavado de ropas y utensilios, y manipulación inadecuada de alimentos que producen una exposición común de la población al virus de la hepatitis $A$ y por lo cual condiciona su transmisión oro-fecal y explican su endemicidad. 
Cuadro 1. Distribución de hepatitis B por categorías y zonas de Riosucio, 1992.

\begin{tabular}{|c|c|c|c|c|c|c|}
\hline \multirow[t]{2}{*}{ Categoría } & \multicolumn{3}{|c|}{$\begin{array}{l}\text { Zona Truandó-Salaquí } \\
\qquad n=458\end{array}$} & \multicolumn{3}{|c|}{$\begin{array}{l}\text { Zona Riosucio-Larga } \\
n=456\end{array}$} \\
\hline & $\mathrm{n}$ & Prevalencia \% & I IC & $\mathbf{n}$ & Prevalencia \% & IC \\
\hline Infección HVB & 419 & 91,5 & $88,4-93,8$ & 33 & 72,4 & $68,0-76,4$ \\
\hline Inmunes & 268 & 64,0 & $59,1-68,5$ & 249 & 54,6 & $49,9-59,2$ \\
\hline Ventana inmunológica & 63 & 15,0 & $11,8-18,9$ & 32 & 7,0 & $4,9-9,8$ \\
\hline Preinmunes & 38 & 9,0 & $6,6-12,3$ & 26 & 5,7 & $3,8-8,4$ \\
\hline Infección reciente & 50 & 11,9 & $9,1-15,5$ & 23 & 5,0 & $3,3-7,4$ \\
\hline
\end{tabular}

Fuente: Padilla J, Arriaga A, Makanaky G. Prevalencia de infección por HVB en Riosucio y riesgos asociados, 1992.

Se ha demostrado que existe una correlación entre la endemicidad de hepatitis A con la higiene y las medidas sanitarias en un área (17).

La región de Truandó-Salaquí, en el municipio de Riosucio, es uno de los focos de hepatitis B y D de más alta prevalencia en el país. La existencia de factores de riesgo como el hacinamiento, la promiscuidad y el inicio de relaciones sexuales en los grupos de 15 a 24 años condicionan la transmisión horizontal y vertical por el virus de la hepatitis viral B; a su vez, la alta frecuencia de portadores de hepatitis $B$ incrementa los riesgos para la transmisión de hepatitis delta por co o sobreinfección, como se ha podido observar en localidades de los municipios de Riosucio y Quibdó.

Las dificultades en la accesibilidad geográfica, económica y cultural, el subdesarrollo de los niveles de atención, la pobre infraestructura diagnóstica, las condiciones de vida de la mayoría de la población chocoana condicionan la endemoepidemicidad de estas enfermedades (18). La carencia de recursos económicos, humanos y materiales son obstáculos para realizar un diagnóstico etiológico de los diferentes tipos de hepatitis y dificultan la confirmación de brotes de la infección. Los esfuerzos iniciales han sido puntuales y difíciles de seguir para obtener resultados a mediano y largo plazo. A su vez, el subdesarrollo de los niveles de atención ensombrece aún más el panorama, siendo necesario plantear soluciones locales para intervenir el problema y buscar el apoyo de entidades a nivel nacional e internacional.

Los resultados encontrados nos muestran que las hepatitis A, B y D representan un grave problema de salud pública en el departamento del Chocó, siendo necesario implementar medidas sostenibles de prevención, vigilancia y control para impactar el problema.

Se recomienda que los servicios de salud y los diferentes sectores involucrados en el problema implementen medidas preventivas enfocadas al mejoramiento de la calidad del agua mediante su tratamiento apropiado, implementar sistemas adecuados de distribución y de eliminación de aguas servidas, educación sanitaria de la población tendiente para lograr un buen saneamiento e higiene personal e insistir en las medidas que reduzcan al mínimo la posibilidad de transmisión fecal-oral de hepatitis $A$.

Igualmente, se requiere implementar programas masivos de vacunación regular contra hepatitis $B$ en población menor de 5 años y establecer sistemas de vigilancia epidemiológica que tengan en cuenta los donantes en bancos de sangre, los grupos sexuales de alto riesgo, los drogadictos y otros, apoyados con pruebas diagnósticas sensibles y específicas $(19,20)$.

En las zonas de alto riesgo de transmisión de hepatitis delta, se debe implementar un sistema de vigilancia activa de síndromes ictéricos apoyados en puestos de viscerotomía.

Es indispensable extremar las medidas de bioseguridad en el personal de salud en riesgo, debido a las altas tasas de infección encontradas; además, se deben realizar programas de tamizaje para detectar susceptibles candidatos a vacunación contra hepatitis B (21).

\section{Agradecimientos}

A Elizabeth Moreno, Víctor Jaime Leguizamón, Augusto Hincapié y Dany Cecilia Vélez, por su valiosa colaboración en la redacción de textos y figuras. A todo el personal del Servicio Seccional 
de Salud del Chocó que colaboraron en la búsqueda de información, especialmente, la Sección de Estadísticas y Epidemiología.

\section{Referencias}

1. Organización Panamericana de la Salud. El control de las enfermedades transmisibles en el hombre. En: Abruma S. Venenos. Washington, D.C: OPS, 1992.

2. Protection againt viral hepatitis. MMWR 1990;39(2).

3. Organización Panamericana de la Salud. La hepatitis en las Americas. Bol Epidemiol 1985;6(5):1-7.

4. Organización Panamericana de la Salud. Hepatitis B y hepatitis delta. Bol Epidemiol 1984;5(3):8.

5. Organización Panamericana de la Salud. Hepatitis viricas en la región. Bol Of Sanit Panam 1986; 100(3):330-7.

6. Buitrago B, Martínez M, Hadler S, et al. Prevalencia de hepatitis A, B y delta en Colombia. Biomédica 1991;11(1-4).

7. Buitrago B. Historia natural de las hepatitis B y D en Colombia. Biomédica 1991;11(1-4).

8. Martínez M, de la Hoz F, Jaramillo L, et al. Seroepidemiología de la infección por el virus de la hepatitis $B$ en niños de la amazonia colombiana. Biomédica 1991;11(1-4).

9. Servicio Seccional de Salud del Chocó Epidemiología de las hepatitis en Chocó. Informe epidemiológico. Quibdó, Chocó, 1992.

10. Servicio Seccional de Salud del Chocó. Enfermedades de notificación obligatoria. Sección de Estadísticas.
11. Engelkes E. Condicionantes epidemiológicos de la morbilidad en el Chocó. Tesis, Quibdó, 1988.

12. Arboleda M, Ja. amillo C, Echeverry M, et al. Un nuevo foco de hepatitis fulminante aguda por virus delta: Urabá, 1987. Bol Epidemiol de Antioquia 1988.

13. Padilla J, Arce N, Makanaky G. Hepatitis fulminante aguda en Bebaramá. Informe de brote. Servisalud Chocó, Quibdó, 1990

14. Padilla J, Arriaga A, Makanaky G. Prevalencia de infección por hepatitis $\mathrm{B}$ y riesgos asociados en Riosucio, 1992: informe preliminar. Bol Epidemiol Chocó 1993;1(1-3).

15. Servicio Seccional de Saiud del Chocó. Informe de brote de la hepatitis fulminante en Guarandó: informe epidemiológico. Quibdó, 1993.

16. Gobernación del Chocó. Plan de desarrollo departamental 1990. Quibdó, 1990.

17. Arriaga A, Arce N, Chamorro A, Padilla J. Prevalencia de marcadores de hepatitis $B$ en trabajadores de la salud en el Chocó. Informe preliminar, Quibdó, 1993.

18. World Health Organization. Public health control of hepatitis A: memorandum from a WHO meeting. Bull World Health Organ 1995;73(1):15-20.

19. Servicio de Salud Chocó. Diagnóstico de la situación de salud en el Chocó. Informe de la jefatura. Quibdó, 1991.

20. Center for Diseases Control and Prevention. Recommendation of the Inmunization Practices Advisory Committe. Inactivated hepatitis B virus vaccine. MMWR 1982;31(24):317-22, 327-9.

21. Hadler SC, Poto L, Maynard JE, et al. Occupational risk of hepatitis infection in hospital workers. Infect Control 1985;6(1):24-31. 